\title{
Versión Transcultural del Cuestionario de Personalidad de Zuckerman- Kuhlman (ZKPQ-50-CC) en Población Argentina
}

\section{Transcultural Version of the Zuckerman Kuhlman Personality Questionnaire (ZKPQ-50-CC) in the Argentinean Population}

\author{
Fernando Martín Poó ${ }^{1,2}$, Rubén Daniel Ledesma ${ }^{1,2}$ y Soledad López ${ }^{2}$ \\ ${ }^{1}$ Consejo Nacional de Investigaciones Científicas y Técnicas de la República Argentina. \\ ${ }^{2}$ Universidad Nacional de Mar del Plata, Argentina.
}

Disponible online 30 de abril de 2013

\begin{abstract}
El objetivo del presente trabajo fue proporcionar evidencia de validez para los puntajes del cuestionario de personalidad ZKPQ-50-CC en población argentina. Este instrumento fue desarrollado simultáneamente en varios países e idiomas, lo que le otorga un especial interés para la investigación transcultural. Se trabajó con una muestra de 895 adultos de población general de la ciudad de Mar del Plata. Se analizaron la validez estructural, la fiabilidad de las escalas y posibles diferencias en los puntajes debidos al género y la edad. Se obtuvo una estructura penta-factorial acorde con el modelo. La estructura replicó la del estudio original. Los niveles de fiabilidad fueron aceptables, y las diferencias de edad y género observadas fueron acordes con la literatura previa. Los hallazgos ofrecen evidencia de validez para el cuestionario ZKPQ-50-CC en un contexto cultural diferente al de los estudios originales. Se requieren estudios de validez complementarios que evalúen otras cualidades psicométricas como la confiabilidad test-re-test, la validez convergente con otras medidas de personalidad y su robustez frente a sesgos de respuesta.
\end{abstract}

Palabras clave: Personalidad; Evaluación; ZKPQ-50-CC; Validez; Fiabilidad.

The aim of this study was to provide evidence of the validity of the ZKPQ-50-CC in the Argentinean population. The ZKPQ-50-CC is a brief personality measure to assess Zuckerman's Alternative Five-Factor Model. This instrument was simultaneously developed in different countries and languages, which makes it of special interest to cross-cultural research. The sample consisted of 895 adults selected from the general population in the city of Mar del Plata (Argentina). Psychometric analysis included dimensional validity, reliability and differences due to gender and age. The results yielded a five-factor structure which was consistent with the model, acceptable degrees of internal consistency, and age and gender differences for the scales. Overall, the results were consistent with the original instrument, although there were some slight differences. The findings provide evidence of the validity of the ZKPQ-50-CC in a cultural context different from those in which the instrument was developed. Further psychometric research is needed to assess the validity of the questionnaire, such as its convergent validity, test-re-test reliability and robustness against social desirability bias.

Key words: Personality; Assessment; ZKPQ-50-CC; Validity; Reliability.

Correspondencia: Fernando Martín Poó, c/ Alberti 4439 Dto 2, Tlf.: 054-0223-4747588; E-mail: poo.fernando@conicet.gov.ar. E-mail de los otros autores: Rubén Daniel Ledesma: rdledesma@conicet.gov.ar; Soledad López: sslopez@mdp.edu.ar

Esta investigación fue realizada con Becas del Consejo Nacional de Investigaciones Científicas y Técnicas (CONICET, Argentina) y subsidios de la Secretaría de Ciencias e Innovación Tecnológica de la Universidad Nacional de Mar del Plata.

Agradecemos al Dr. Anton Aluja por habernos brindado la matriz factorial del estudio original del ZKPQ-50-CC. 
Aluja et al. (2006) desarrollaron el ZKPQ-50-CC, una versión transcultural breve del cuestionario ZKPQ-III-R (Zuckerman, Kuhlman, Joireman, Teta y Kraft, 1993) que evalúa el modelo de personalidad biológico factorial de los Cinco Alternativos (Zuckerman, Kuhlman, Thornquist, \& Kiers, 1991). Zuckerman (2005) brinda numerosa evidencia acerca de las bases genéticas, psicofisiológicas y psiconeurológicas de los rasgos que conforman su modelo. Estos rasgos son: (1) Impulsividad-Búsqueda de Sensaciones, (2) Neuroticismo-Ansiedad, (3) Agresión-Hostilidad, (4) Actividad, y (5) Sociabilidad.

El ZKPQ-50-CC fue elaborado al mismo tiempo en cuatro idiomas, castellano (España), francés (Suiza), alemán (Alemania) e inglés (Estados Unidos). Conservó la misma estructura factorial en los cuatro idiomas en los que se desarrolló y replicó la del instrumento original.

Todos los países en los que se construyó y validó el ZKPQ50-CC poseen cierta similitud en cuanto a su nivel de desarrollo, al menos si se los compara con países latinoamericanos como Argentina. Todos estos países pertenecen al hemisferio norte y poseen economías de altos ingresos a diferencia de Argentina que es un país de medianos ingresos. Por otra parte, aunque en este país predomina el elemento étnico europeo, su población se conformó a través de numerosas oleadas de inmigrantes que se mestizaron entre sí y con los pueblos originarios. Estos procesos constituyeron una cultura particular y distintiva. En consecuencia, cabe preguntarse si los resultados informados en el estudio original son replicables en esta población.

El objetivo del presente trabajo fue analizar las características psicométricas del ZKPQ-50-CC en población general de adultos argentinos. Se buscó aportar evidencia de validez para el instrumento, y ampliar el alcance de la investigación transcultural realizada a la fecha. Específicamente, se quiso conocer la estructura factorial del instrumento, la consistencia interna de sus escalas y posibles diferencias atribuibles al género y la edad.

\section{Método \\ Participantes}

Se empleó una muestra no probabilística constituida por 895 individuos de la población general de la ciudad de Mar del Plata. El rango de edad de los participantes fue de 18 a 87 años $(M=36.38 ; D T=13.58)$. El $42 \%$ de los participantes fueron mujeres y el $58 \%$ restante fueron hombres. La muestra estuvo conformada por empleados públicos y privados (38.1\%); trabajadores autónomos y profesionales (35.8\%); estudiantes $(8.1 \%)$, retirados y amas de casa $(18 \%)$. La mayoría de los participantes $(80.6 \%)$ había completado la escuela secundaria al momento del estudio.

\section{Instrumentos}

Los ítems de la versión en castellano del ZKPQ-50-CC fueron revisados para evaluar su adecuación con el habla colo- quial en la Argentina. Como resultado, dieciocho ítems fueron modificados levemente. En ningún caso se alteró el sentido de las afirmaciones. El cuestionario tiene un formato de respuesta dicotómico (verdadero-falso). Además del ZKPQ-50-CC se administró un cuestionario sobre aspectos sociodemográficos (género, edad, nivel educativo y ocupación).

\section{Procedimiento}

Se utilizó un muestreo casual. Los participantes fueron contactados en diferentes lugares públicos y fueron invitados a participar del estudio de manera individual y voluntaria. No recibieron ninguna recompensa a cambio de su participación. Se garantizó el anonimato de las respuestas y la confidencialidad en el tratamiento de los datos. Los participantes brindaron su consentimiento informado. Completar el cuestionario tomó aproximadamente 10 minutos. Un total de 124 personas no accedieron a participar del estudio.

Análisis de datos. Por tratarse de ítems binarios, se aplicó análisis factorial exploratorio sobre la matriz de correlaciones tetracóricas. De acuerdo con las dimensiones del modelo de los Cinco Alternativos, en primer lugar se solicitaron cinco factores con rotación oblicua. En todos los casos se obtuvieron correlaciones entre factores inferiores a 0.20. Por lo tanto, se utilizó rotación ortogonal. Se estimó además el ajuste global de la solución pentafactorial (Ferrando \& Anguiano-Carrasco, 2010). Se calcularon estadísticos descriptivos y coeficientes de fiabilidad (Alfa de Cronbach) para cada una de las escalas resultantes. Se analizaron las posibles diferencias atribuibles al género y a la edad mediante análisis multivariado de la varianza (MANOVA). Para los análisis se definieron dos grupos de edad: jóvenes (hasta 30 años) y adultos (mayores de 30 años). Al respecto, existe numerosa evidencia que indica que la estructura de la personalidad de un individuo adquiere su madurez alrededor de los 30 años y que luego de esa edad sólo ocurren pequeños y graduales cambios (Costa \& McCrae, 2006; McCrae \& Costa, 1999). Si bien existen autores que discuten estos resultados (e.g. Roberts \& Mroczek, 2008) consideramos que el criterio utilizado para segmentar la muestra es coherente con los objetivos del presente estudio.

Los datos fueron gestionados y analizados con los programas Factor (Lorenzo-Seva \& Ferrando, 2006), Vista 6.4 (Young, 1996), Coeficient of Congruence (Watkins, 2012), y SPSS 11.5.

\section{Resultados}

El análisis factorial indicó la existencia de cinco factores acordes a las dimensiones del ZKPQ-50-CC (ver tabla 1). La varianza total explicada fue del $46 \%$, y el ajuste del modelo pentafactorial resultó satisfactorio (GFI: .95). El primer factor explicó el $13.5 \%$ de la varianza total y corresponde a la escala Agresión-Hostilidad. El segundo factor explicó el 11.8 \% de la varianza total y está compuesto por ítems que pertenecen a la 
Tabla 1

Cargas factoriales, Medias, D.E., e índices de discriminación para los ítems del ZKPQ-50-CC $(n=895)$

\begin{tabular}{|c|c|c|c|c|c|c|c|c|}
\hline \multirow[t]{2}{*}{ Items $^{(a)}$} & \multicolumn{5}{|c|}{ Carga Factorial } & \multirow[t]{2}{*}{$M$} & \multirow[t]{2}{*}{$D T$} & \multirow[t]{2}{*}{$D^{(b)}$} \\
\hline & AggHost & N-Anx & Sy & ImpSS & Act & & & \\
\hline 44. & -.73 & .04 & -.03 & -.05 & -.16 & .38 & .48 & .48 \\
\hline 37. & .66 & .04 & -.15 & .18 & -.05 & .40 & .49 & .48 \\
\hline 50. & .65 & .08 & -.07 & .11 & -.08 & .41 & .49 & .48 \\
\hline 3. & .64 & .02 & .11 & .14 & -.01 & .55 & .49 & .49 \\
\hline 35. & .61 & -.10 & -.02 & .21 & .09 & .48 & .50 & .46 \\
\hline 2. & .55 & -.16 & -.00 & .18 & -.03 & .73 & .44 & .40 \\
\hline 7. & -.50 & .18 & .06 & .03 & -.10 & .25 & .43 & .37 \\
\hline 32. & .49 & .11 & -.16 & .21 & .01 & .41 & .49 & .39 \\
\hline 11. & -.46 & .22 & .15 & -.13 & -.08 & .39 & .48 & .25 \\
\hline 19. & .28 & -.24 & .03 & .24 & .03 & .65 & .48 & .22 \\
\hline 22. & .06 & .81 & -.00 & .02 & -.01 & .09 & .29 & .45 \\
\hline 18. & -.03 & .78 & -.02 & -.00 & -.02 & .13 & .34 & .45 \\
\hline 10. & .04 & .63 & -.04 & .15 & .00 & .19 & .39 & .39 \\
\hline 40. & .03 & .61 & .05 & -.01 & .00 & .11 & .31 & .33 \\
\hline 14. & .14 & .54 & -.01 & -.04 & .03 & .44 & .49 & .36 \\
\hline 46. & .24 & .52 & -.21 & .10 & .00 & .31 & .46 & .40 \\
\hline 27. & -.11 & .52 & -.24 & .07 & -.15 & .38 & .48 & .35 \\
\hline 9. & .10 & .39 & -.25 & -.01 & .07 & .49 & .50 & .32 \\
\hline 43. & .42 & -.33 & .01 & -.21 & .00 & .33 & .47 & .24 \\
\hline 31. & -.03 & .20 & -.11 & .08 & .20 & .67 & .47 & .21 \\
\hline 33. & .04 & -.12 & -.72 & .01 & .01 & .48 & .50 & .54 \\
\hline 45. & -.02 & -.10 & -.68 & .04 & -.08 & .45 & .49 & .51 \\
\hline 23. & .05 & -.06 & .70 & .20 & .13 & .47 & .49 & .46 \\
\hline 20. & -.06 & .29 & -.58 & .22 & .07 & .62 & .48 & .47 \\
\hline 28. & -.08 & -.03 & -.47 & .19 & .03 & .43 & .49 & .30 \\
\hline 15. & .04 & .15 & -.45 & .03 & .07 & .26 & .44 & .26 \\
\hline 8. & .02 & -.15 & .42 & .31 & .00 & .59 & .49 & .34 \\
\hline 47. & .02 & .10 & .40 & .35 & .00 & .29 & .45 & .31 \\
\hline 38. & -.31 & -.18 & .36 & .23 & .23 & .70 & .45 & .36 \\
\hline 4. & -.10 & -.19 & -.28 & .03 & -.12 & .66 & .47 & .28 \\
\hline 34. & .28 & .17 & .16 & .62 & .10 & .22 & .41 & .50 \\
\hline 24. & .29 & -.01 & .14 & .58 & .03 & .51 & .50 & .49 \\
\hline 26. & .05 & -.03 & -.12 & .55 & -.05 & .44 & .49 & .26 \\
\hline 42. & .24 & .20 & .23 & .54 & .15 & .37 & .48 & .48 \\
\hline 13. & -.02 & -.04 & .02 & .49 & -.05 & .72 & .44 & .26 \\
\hline 17. & .26 & .04 & .31 & .48 & .09 & .48 & .50 & .48 \\
\hline 30. & .09 & .05 & .26 & .42 & .24 & .62 & .48 & .38 \\
\hline 6. & .50 & .23 & .09 & .42 & .09 & .51 & .50 & .39 \\
\hline 49. & .15 & .00 & .61 & .41 & .05 & .52 & .49 & .39 \\
\hline 39. & .11 & .34 & .24 & .27 & .18 & .22 & .41 & .27 \\
\hline 41. & -.11 & .34 & .24 & .26 & .89 & .52 & .49 & .64 \\
\hline 12. & -.13 & -.08 & -.00 & -.06 & .84 & .59 & .49 & .61 \\
\hline 36. & -.18 & -.09 & -.05 & -.09 & .73 & .64 & .47 & .51 \\
\hline 21. & .23 & .18 & .23 & -.19 & -.72 & .35 & .47 & .49 \\
\hline 1. & -.12 & -.07 & -.10 & .02 & .61 & .48 & .50 & .43 \\
\hline 16. & .20 & .17 & .20 & .25 & -.57 & .28 & .45 & .38 \\
\hline 29. & .13 & .21 & .12 & .12 & .49 & .32 & .47 & .35 \\
\hline 25. & -.06 & -.10 & .23 & .18 & .38 & .40 & .49 & .28 \\
\hline 48. & -.15 & -.29 & -.08 & .08 & .36 & .81 & .38 & .21 \\
\hline 5. & -.09 & -.05 & -.28 & .09 & .27 & .46 & .49 & .16 \\
\hline
\end{tabular}

Nota: Agg-Host: Agresión-Hostilidad, N-Anx: Neuroticismo-Ansiedad, Sy: Sociabilidad, Imp-SS: Impulsividad-Búsqueda de Sensaciones, Act: Actividad

Extracción: ULS (Mínimos cuadrados no ponderados). Rotación: Varimax

a. Número de ítems de acuerdo con la versión original del ZKPQ-50$\mathrm{CC}$

b. Discriminación del ítem: Correlación item-total corregida. escala Neuroticismo-Ansiedad. El tercer factor explicó el $8.9 \%$ de la varianza total. Está compuesto por ítems que pertenecen en su totalidad a la escala Sociabilidad. El cuarto factor corresponde a la escala Impulsividad-Búsqueda de Sensaciones y explicó el $6.6 \%$ de la varianza total. Por último, el quinto factor explicó el $5.1 \%$ de la varianza total y está compuesto por ítems que pertenecen a la escala Actividad.

Excepto cinco ítems, todos mostraron cargas superiores a .30 en sus respectivos factores. Los ítems con cargas ligeramente menores fueron el 19 , el 31 , el 4 , el 39 y el 5 . Todos cargaron en los factores esperados de acuerdo con la versión original del instrumento, por lo tanto, se conservó a cada ítem en su respectiva escala.

Los índices de congruencia factorial entre las escalas de la versión argentina y española fueron: Agresión-Hostilidad $=.97$, Ansiedad-Neuroticismo $=.94$, Sociabilidad $=.96, \mathrm{Im}-$ pulsividad-Búsqueda de Sensaciones $=.97$ y Actividad $=.98$. El índice de congruencia total fue .98. Los valores indican una buena replicabilidad del modelo de los Cinco Alternativos en población argentina.

Tabla 2

Estadísticos descriptivos, consistencia interna y correlaciones con la edad para las escalas del ZKPQ-50-CC. Comparación con los resultados informados por Aluja et al. (2006).

\begin{tabular}{|c|c|c|c|c|c|c|c|}
\hline & & M & $D T$ & Asimetría & Curtosis & $\begin{array}{l}\text { Alfa de } \\
\text { Cronbach }\end{array}$ & $\begin{array}{l}\text { Correlación } \\
\text { con la edad }\end{array}$ \\
\hline Argentina & N-Anx & 3.20 & 2.19 & .72 & 0.46 & .68 & $.03^{\mathrm{n} . \mathrm{s}}$ \\
\hline \multirow[t]{4}{*}{$(n=895)$} & ImpSS & 4.65 & 2.58 & .24 & -0.77 & .74 & $-.28 * * *$ \\
\hline & Act & 4.90 & 2.60 & -.13 & -0.98 & .76 & $.09 * *$ \\
\hline & Sy & 5.00 & 2.58 & .01 & -0.82 & .73 & $-.23 * * *$ \\
\hline & Agg-Host & 4.68 & 2.64 & .19 & -0.89 & .74 & $-.11 * * *$ \\
\hline EUA & N-Anx & 5.16 & 2.50 & -.03 & -1.11 & .80 & -- \\
\hline \multirow[t]{4}{*}{$(n=2.378)$} & ImpSS & 5.94 & 2.16 & -.28 & -0.68 & .72 & -- \\
\hline & Act & 4.15 & 2.60 & .31 & -0.82 & .74 & -- \\
\hline & Sy & 6.46 & 2.53 & -.62 & -0.35 & .74 & -- \\
\hline & Agg-Host & 5.47 & 2.56 & -.11 & -0.83 & .72 & -- \\
\hline Alemania & N-Anx & 4.77 & 2.52 & .11 & -1.15 & .82 & $-.14 * * *$ \\
\hline \multirow[t]{4}{*}{$(n=517)$} & ImpSS & 4.19 & 2.24 & .28 & -0.62 & .73 & $-.19 * * *$ \\
\hline & Act & 4.81 & 2.77 & .11 & -0.97 & .74 & $-.09^{*}$ \\
\hline & Sy & 5.28 & 2.34 & -.35 & -0.83 & .67 & $-.13^{* *}$ \\
\hline & Agg-Host & 4.79 & 2.30 & .23 & -0.64 & .60 & $-.12 * *$ \\
\hline España & N-Anx & 4.18 & 2.23 & .51 & -0.73 & .79 & $-.16^{* * *}$ \\
\hline \multirow[t]{4}{*}{$(n=962)$} & ImpSS & 5.27 & 2.35 & -.29 & -.80 & .73 & $-.14 * * *$ \\
\hline & Act & 4.89 & 2.84 & -.04 & -1.10 & .79 & $-.03 \mathrm{n} . \mathrm{s}$ \\
\hline & Sy & 6.51 & 2.49 & -.60 & -0.42 & .74 & $-.12 * * *$ \\
\hline & Agg-Host & 4.86 & 2.42 & .10 & -0.75 & .66 & $-.07 *$ \\
\hline Suiza & N-Anx & 4.87 & 2.62 & .18 & -1.08 & .83 & $-.11 * *$ \\
\hline \multirow[t]{4}{*}{$(n=764)$} & ImpSS & 5.38 & 2.33 & -.16 & -0.79 & .74 & $-.08^{*}$ \\
\hline & Act & 3.76 & 2.60 & .44 & -0.75 & .76 & $-.01 \mathrm{n} . \mathrm{s}$ \\
\hline & Sy & 5.67 & 2.42 & -.89 & -0.69 & .71 & $-.08^{*}$ \\
\hline & Agg-Host & 4.72 & 2.44 & .06 & -0.82 & .68 & $-.03 \mathrm{n} . \mathrm{s}$ \\
\hline
\end{tabular}

Nota: ${ }^{*} p<.05$ bilateral. ${ }^{* *} p<.01$ bilateral. ${ }^{* * *} p<.001 . \mathrm{n} . \mathrm{s}=$ no significativo

Agg-Host: Agresión-Hostilidad. N-Anx: Neuroticismo-Ansiedad. Sy: Sociabilidad. Imp-SS: Impulsividad-Búsqueda de Sensaciones. Act: Actividad. 
La Tabla 2 muestra los estadísticos descriptivos para las escalas en comparación con los resultados obtenidos en el estudio transcultural de Aluja et al. (2006). Globalmente, los resultados en Argentina tienden a ser similares, tanto a nivel descriptivo como en el patrón de correlaciones con la edad. No obstante, se advierten ciertas diferencias en la escala NeuroticismoAnsiedad. Esta escala obtiene una media comparativa menor, y también un valor de consistencia interna más bajo que en el resto de los países. Un dato interesante es que en la muestra de hombres el Alfa de Cronbach es bastante menor que en la muestra de mujeres (.63, y .71 respectivamente). La escala tampoco se relacionó negativamente con la edad, como se observa en el resto de los países.

Un análisis multivariado (MANOVA) reveló diferencias significativas por género, $F(5,884)=21.481, p<.001, \eta^{2}$ parcial $=.11$, y por edad $F(5,884)=18.206, p<.001, \eta^{2}$ parcial $=.09$. En el caso del género, el ANOVA univariado indicó diferencias en las escalas de Agresión-Hostilidad, $F(1,888)=20.243$, $p<.001, \eta^{2}$ parcial $=.02$; Impulsividad-Búsqueda de Sensaciones, $F(1,888)=16.531, p<.001, \eta^{2}$ parcial $=.02$; Sociabilidad, $F(1,888)=16.949, p<.001, \eta^{2}$ parcial $=.02 ;$ y NeuroticismoAnsiedad, $F(1,888)=22.364, p<.001, \eta^{2}$ parcial $=.02$. No se observaron diferencias significativas para la escala Actividad $[F(1,888)=.004, p>.05]$. Las puntuaciones medias para las escalas del ZKPQ de acuerdo con el género pueden observarse en la Tabla 3. En el caso de la edad, el ANOVA univariado reveló diferencias en las escalas de Agresión-Hostilidad, $F(1,888)=$ $6.288, p<.05, \eta^{2}$ parcial $=.01$; Actividad, $F(1,888)=16.275, p<$ $.001, \eta^{2}$ parcial $=.02$; Impulsividad-Búsqueda de Sensaciones, $F(1,888)=44.384, p<.001, \eta^{2}$ parcial $=.05 ;$ y Sociabilidad, $F(1,888)=31.949, p<.001, \eta^{2}$ parcial $=.03$. No se observaron diferencias significativas para la escala Neuroticismo-Ansiedad $[F(1,888)=1.959, p>.05]$. Las puntuaciones medias para las escalas según grupos de edad pueden verse en la Tabla 3. Sintéticamente, los jóvenes obtuvieron puntajes más elevados en las escalas Agresión-Hostilidad, Impulsividad-Búsqueda de Sensaciones, y Sociabilidad, mientras que los adultos puntuaron más en la escala Actividad.

Tabla 3

Medias. Desvíos Estándar y puntajes $t$ para las escalas del ZKPQ-50CC diferenciadas de acuerdo con el género

\begin{tabular}{|c|c|c|c|c|c|c|c|c|c|c|}
\hline \multirow{2}{*}{ Escalas } & \multicolumn{2}{|c|}{ Hombres } & \multicolumn{2}{|c|}{ Mujeres } & \multirow[t]{2}{*}{$t$} & \multirow[t]{2}{*}{$d$} & \multicolumn{2}{|c|}{ Jóvenes } & \multicolumn{2}{|c|}{ Adultos } \\
\hline & $M$ & $D T$ & $M$ & $D T$ & & & $M$ & $D T$ & $M$ & $D T$ \\
\hline Aggr-Host & 5.02 & 2.65 & 4.22 & 2.55 & $4.55^{* * *}$ & 0.31 & 4.93 & 2.67 & 4.50 & 2.60 \\
\hline N-Anx & 2.89 & 2.00 & 3.59 & 2.37 & $-4.82 * * *$ & -0.32 & 4.52 & 2.64 & 5.18 & 2.58 \\
\hline Sy & 4.68 & 2.51 & 5.43 & 2.62 & $-4.32 * * *$ & -0.29 & 5.31 & 2.66 & 4.14 & 2.41 \\
\hline Imp-SS & 4.92 & 2.54 & 4.27 & 2.60 & $3.72 * * *$ & 0.25 & 5.58 & 2.56 & 4.56 & 2.51 \\
\hline Act & 4.87 & 2.58 & 4.93 & 2.68 & $0.32 \mathrm{n} . \mathrm{s}$ & -0.02 & 3.08 & 2.21 & 3.27 & 2.17 \\
\hline ota: * & & & & & & & & & & \\
\hline
\end{tabular}

\section{Discusión}

Los resultados ofrecen evidencia de validez para los puntajes del ZKPQ-50-CC en población argentina. El análisis factorial mostró una estructura consistente con la obtenida por Aluja et al. (2006) y acorde con lo esperado teóricamente (Zuckerman, 2005). La composición de las dimensiones fue la misma que la del estudio original, y las escalas mostraron coeficientes de consistencia interna aceptables.

Las diferencias por género y edad fueron similares a lo informado en estudios previos con distintas versiones del ZKPQ. Sin embargo, los tamaños del efecto fueron de moderados a bajos en la mayoría de los casos (Cohen, 1988). En cuanto al género los resultados tienden a ser consistentes al señalar que los hombres obtienen puntuaciones más elevadas en Impulsividad-Búsqueda de Sensaciones y Agresión-Hostilidad, mientras que las mujeres los obtienen en Neuroticismo-Ansiedad y Sociabilidad (Aluja et al., 2006; Goma i Freixanet \& Valero Ventura, 2008; Zuckerman et al. 1993). Por otro lado, el análisis de las diferencias de edad también resultó acorde al trabajo original de Aluja et al. (2006). Los individuos más jóvenes tienden a obtener puntuaciones más elevadas en ImpulsividadBúsqueda de Sensaciones, Agresión-Hostilidad y Sociabilidad, y menores en Actividad. Aluja et al. (2006) también observaron una relación leve y negativa entre la edad y la escala de Neuroticismo-Ansiedad en las distintas muestras de validación del ZKPQ-50-CC, pero nuestros datos no acompañan esta tendencia. Finalmente, resulta interesante señalar que el rango de edad de la muestra del presente estudio fue más amplio que el de los estudios previos.

En síntesis, creemos que los resultados del estudio amplían la evidencia de validez para los puntajes del ZKPQ-50-CC. A pesar del carácter transcultural del cuestionario, la información a la fecha se limitaba a los países participantes del estudio original. Por otro lado, aunque los resultados fueron globalmente satisfactorios, se requieren estudios de validez complementarios que evalúen otras cualidades psicométricas, como la confiabilidad test-re-test, la validez convergente con otras medidas de personalidad y su robustez frente a posibles sesgos de respuesta.

\section{Referencias}

1. Aluja, A., Rossier, J., García L. F., Angleitner, A., Kuhlman, M. \& Zuckerman, M. (2006). A cross-cultural shortened form of the ZKPQ (ZKPQ-50-CC) adapted to English, French, German, and Spanish languages. Personality and Individual Differences, 41, 619-628. http://dx.doi. org/10.1016/j.paid.2006.03.001

2. Cohen, J. (1988). Statistical power analysis for the behavior sciences (2nd ed) (p.283). New Jersey. Lawrence Erlbaum.

3. Costa, P.T. \& McCrae, R.R. (2006). Age changes in personality and their origins: Comment on Roberts, Walton, and Viechtbauer. Psychological Bulletin, 132, 26-28. http:// dx.doi.org/10.1037/0033-2909.132.1.26 
4. Ferrando, P. J. \& Anguiano-Carrasco, C. (2010). El análisis factorial como técnica de investigación en psicología. $P a-$ peles del Psicólogo, 31, 18-33.

5. Goma i Freixanet, M. \& Valero Ventura, S. (2008). Spanish normative data of the Zuckerman-Kuhlman Personality Questionnaire in a general population sample. Psicothema, 20, 324-330.

6. Lorenzo-Seva, U. \& Ferrando, P. (2006). Manual of the program factor. Extraído el 12 de septiembre de 2011 de la página web: http://psico.fcep.urv.es/utilitats/factor/

7. McCrae, R.R. \& Costa, P.T. (1999). A Five-Factor Theory of personality. In L.A. Pervin and O.P John (Eds.), Handbook of personality: Theory and research (pp. 139 -153). New York: Guilford Press.

8. Roberts, B. W. \& Mroczek, D (2008) Personality trait change in adulthood. Current directions in Psychological Science, 17, 31-35. http://dx.doi.org/10.1111/j.1467$\underline{8721.2008 .00543 . \mathrm{x}}$

9. Watkins, M. (2012). Coeficiente of Congruence. http://edpsychassociates.com/Watkins3.html

10. Young, F. W. (1996). ViSta "The Visual Statistics System" [Software]. http://forrest.psych.unc.edu/research/index. html
11. Zuckerman, M (2005). Psychobiology of personality. Cambridge: Cambridge University Press. http://dx.doi. org/10.1017/CBO9780511813733

12. Zuckerman, M., Kuhlman, D. M., Joireman, J., Teta, P. \& Michael Kraft, M. (1993). A Comparison of Three Structural Models for Personality: The Big Three, the Big Five, and the Alternative Five. Journal of Personality and Social Psychology, 65, 757-768. http://dx.doi.org/10.1037/0022$\underline{3514.65 .4 .757}$

13. Zuckerman, M., Kuhlman, D. M., Thornquist, M., \& Kiers, H. (1991). Five (or three) robust questionnaire scale factors of personality without culture. Personality and Individual Diferences, 12, 929-941. http://dx.doi.org/10.1016/01918869(91)90182-B

Fecha de recepción: 9 de febrero de 2012

Fecha de recepción de la primera versión modificada: 29 de junio de 2012

Fecha de recepción de la segunda versión modificada: 6 de agosto de 2012 Fecha de aceptación: 6 de septiembre de 2012 